\title{
The Phosphodiesterase Inhibitor IBMX Blocks the Potassium Channel THIK-1 from the Extracellular Side
}

\author{
Xinle Zou, ${ }^{1}$ Linus J. Conrad, ${ }^{1}$ Kristin Koschinsky, Günter Schlichthörl, Regina Preisig-Müller, \\ Eugen Netz, Jens Krüger, Jürgen Daut, and Vijay Renigunta \\ Institute of Physiology and Pathophysiology, Marburg University, Marburg, Germany (X.Z., L.J.C., K.K., G.S., R.P.-M., J.D., V.R.); \\ Biomolecular Interactions, Max Planck Institute for Developmental Biology, Tübingen, Germany (E.N.); and High Performance \\ and Cloud Computing Group, IT Center, University of Tübingen, Tübingen, Germany (J.K.)
}

Received March 17, 2020; accepted April 16, 2020

\section{ABSTRACT}

The two-pore domain potassium channel ( $\mathrm{K}_{2 \mathrm{P}}$-channel) THIK-1 has several predicted protein kinase $A(P K A)$ phosphorylation sites. In trying to elucidate whether THIK-1 is regulated via PKA, we expressed THIK-1 channels in a mammalian cell line $(\mathrm{CHO}$ cells) and used the phosphodiesterase inhibitor 3-isobutyl-1methyl-xanthine (IBMX) as a pharmacological tool to induce activation of PKA. Using the whole-cell patch-clamp recording, we found that THIK-1 currents were inhibited by application of IBMX with an $\mathrm{IC}_{50}$ of $120 \mu \mathrm{M}$. Surprisingly, intracellular application of IBMX or of the second messenger CAMP via the patch pipette had no effect on THIK-1 currents. In contrast, extracellular application of IBMX produced a rapid and reversible inhibition of THIK-1. In patch-clamp experiments with outsideout patches, THIK-1 currents were also inhibited by extracellular application of IBMX. Expression of THIK-1 channels in Xenopus oocytes was used to compare wild-type channels with mutated channels. Mutation of the putative PKA phosphorylation sites did not change the inhibitory effect of IBMX on THIK-1 currents. Mutational analysis of all residues of the (extracellular) helical cap of THIK-1 showed that mutation of the arginine residue at position 92 , which is in the linker between cap helix 2 and pore helix 1, markedly reduced the inhibitory effect of IBMX. This flexible linker region, which is unique for each $\mathrm{K}_{2 \mathrm{P}}$-channel subtype, may be a possible target of channelspecific blockers.

\section{SIGNIFICANCE STATEMENT}

The potassium channel THIK-1 is strongly expressed in the central nervous system. We studied the effect of 3-isobutyl-1methyl-xanthine (IBMX) on THIK-1 currents. IBMX inhibits breakdown of cAMP and thus activates protein kinase $A$ (PKA). Surprisingly, THIK-1 current was inhibited when IBMX was applied from the extracellular side of the membrane, but not from the intracellular side. Our results suggest that IBMX binds directly to the channel and that the inhibition of THIK-1 current was not related to activation of PKA.

\section{Introduction}

There are about 80 potassium channel subunits in the human genome ( $\mathrm{Yu}$ and Catterall, 2004). These channels regulate the function of many cell types by three basic mechanisms: 1) $\mathrm{K}^{+}$channels are responsible for the repolarization process of action potentials. 2 ) $\mathrm{K}^{+}$channels modulate the excitability by setting the resting membrane potential. 3) By modulating the membrane potential, $\mathrm{K}^{+}$channels indirectly control the influx of calcium ions through voltageactivated $\mathrm{Ca}^{2+}$ channels and transient-receptor-potential channels, which influences many cellular processes. So far,

This study was supported by the Deutsche Forschungsgemeinschaft to J.D. [Grants FOR 1086 TP7; SFB 593, TP4] and to J.K. [Grant INST 37/935-1 FUGG].

${ }^{1}$ X.Z. and L.J.C. contributed equally to this work.

https://doi.org/10.1124/mol.120.000011. it has been difficult to develop blockers that specifically block only one $\mathrm{K}^{+}$channel or only a particular subfamily of $\mathrm{K}^{+}$ channels. This can be explained by the fact that most channel blockers target the central cavity of the channel, which is accessible from the inside of the cell. Because the dimensions and the inner walls of the channels are quite well conserved, most drugs inhibit several different potassium channels to some extent.

The family of two-pore domain $\mathrm{K}^{+}$channels $\left(\mathrm{K}_{2 \mathrm{P}}\right.$-channels) is unique among potassium channels in that they form dimers, with each subunit contributing two pore helices to the transmembrane pore. $\mathrm{K}_{2 \mathrm{P}}$-channels also possess a unique extracellular "helical cap" structure that allows the efflux of potassium ions into the extracellular space through two lateral openings (Brohawn et al., 2012; Miller and Long, 2012). This structural peculiarity may allow the development of drugs that specifically target $\mathrm{K}_{2 \mathrm{P}}$-channels. Since the helical caps of different

ABBREVIATIONS: $\tau_{\text {on }}$, time constant of the binding; $\tau_{\text {off }}$, dissociation time constant; $C 2$, cap helix 2 ; cRNA, complementary RNA; $h$, human; $I_{0}$, current measured immediately before application of IBMX; IBMX, 3-isobutyl-1-methyl-xanthine; $I_{\mathrm{BBMX}}$, current measured in the steady state after application of IBMX; $I_{\mathrm{IBMX}} / \mathrm{I}_{0}$, fractional residual current measured in the presence of IBMX; $\mathrm{K}_{2 \mathrm{P}}$-channel, two-pore domain $\mathrm{K}^{+}$channel; P1, pore helix 1; PKA, protein kinase A; $r$, rat; TASK-3, TWIK-related acid-sensitive $\mathrm{K}+$ channel 3; THIK-1, tandem pore domain halothane inhibited $\mathrm{K}^{+}$ channel 1; THIK-2, tandem pore domain halothane inhibited $\mathrm{K}^{+}$channel 2; TPA, tetrapentylammonium; TRAAK, TWIK-related arachidonic acid-stimulated $\mathrm{K}^{+}$channel; TREK, TWIK-related $\mathrm{K}^{+}$channel; TWIK, tandem of $\mathrm{P}$ domains in a weakly inwardly rectifying $\mathrm{K}^{+}$channel. 
$\mathrm{K}_{2 \mathrm{P}}$-channels show only moderate homology, it may even be possible to develop tailored drugs that are specific for one particular $\mathrm{K}_{2 \mathrm{P}}$-channel subtype.

In humans, the family of $\mathrm{K}_{2 \mathrm{P}}$-channels has 15 members (Enyedi and Czirják, 2010). Most of the $\mathrm{K}_{2 \mathrm{P}}$-channels are characterized by a very complex regulation of open probability (Feliciangeli et al., 2015; Renigunta et al., 2015), and the function of various $\mathrm{K}_{2 \mathrm{P}}$-channel subtypes in the brain, heart, and endocrine glands has been characterized in some detail (Duprat et al., 2007; Bayliss and Barrett, 2008; Lesage and Barhanin, 2011; Bichet et al., 2015). Tandem pore domain halothane-inhibited $\mathrm{K}^{+}$channel 1 (THIK-1) and THIK-2 represent a $\mathrm{K}_{2 \mathrm{P}}$-channel subfamily that has received relatively little attention so far (Rajan et al., 2001; Chatelain et al., 2013; Renigunta et al., 2014). A distinguishing feature of these channels is their inhibition by the inhalational anesthetics halothane and isoflurane (Rajan et al., 2001; Lazarenko et al., 2010; Chatelain et al., 2013; Kang et al., 2014; Renigunta et al., 2014). Reverse-transcription polymerase chain reaction analysis showed that THIK-1 is expressed ubiquitously both in the adult and during embryogenesis (Rajan et al., 2001; Aller and Wisden, 2008). In situ hybridization experiments showed that THIK-1 is robustly expressed in rat brain, especially in some thalamic nuclei, the dentate gyrus, the ventromedial hypothalamic nucleus, and the olfactory bulb (Girard et al., 2001; Rajan et al., 2001). The function of THIK-1 and THIK-2 channels in the brain is still unclear. Interestingly, THIK-1 channels have recently been shown to be strongly expressed in microglial cells (Butovsky et al., 2014; Zhang et al., 2014) and to play an essential role in controlling the morphology and the immunologic function of these cells (Madry et al., 2018).

The present study was initiated by a serendipitous finding. When studying the signal transduction between G-protein-coupled receptors and THIK-1 channels in heterologous expression systems, we found that application of the phosphodiesterase inhibitor 3-isobutyl-1-methyl-xanthine (IBMX) rapidly and reversibly inhibited THIK-1 currents. Investigation of the underlying mechanism showed that THIK-1 channels were insensitive to intracellular application of IBMX and that mutation of a residue of the extracellular cap (R92) reduced the sensitivity to extracellular application of IBMX. Taken together, our results suggest that the effect of IBMX was not caused by inhibition of an enzyme but by direct binding to the extracellular cap of THIK-1.

\section{Methods}

Ethical Approval. For experiments involving Xenopus oocytes, adult female African clawed frogs (Xenopus laevis) were used. The frogs were anesthetized by putting them in water containing $1 \mathrm{~g} / \mathrm{l}$ tricaine. Stage-V oocytes were obtained from ovarian lobes. Anesthesia and operation were carried out in accordance with the principles of German legislation with approval of the animal welfare officer of the Medical Faculty of Marburg University under the governance of the Regierungspräsidium Giessen (the regional veterinary health authority).

Molecular Cloning and Mutagenesis. Full-length rat THIK-1 (accession number NM 022293), human TREK-1c (accession number AY552980), and human TWIK-related arachidonic acid-stimulated $\mathrm{K}^{+}$channel (TRAAK, accession number AF247042) channels were cloned into the pSGEM oocyte expression vector and into the mammalian expression vector pCDNA3.1 (Rajan et al., 2001; Renigunta et al., 2014; Rinné et al., 2014). The QuikChange site-directed mutagenesis kit (Agilent Technologies) was used to introduce point mutations. All constructs were verified by sequencing.

Patch-Clamp Measurements of THIK Currents in Transfected CHO Cells. CHO cells were cultured in Eagle's Minimum Essential Medium- $\alpha$ with glutamine (Thermo Fisher), 10\% Fetal Calf Serum, and 1\% penicillin/streptomycin. The cells were seeded in 35-mm glass-bottom dishes (ibidi, Martinsried, Germany) and were transfected with either rat THIK-1, human TREK-1c, hTRAAK, or hTREK-1 $\mathrm{c}^{\mathrm{S} 344 \mathrm{~A}}$ cloned into the pCDNA3.1 mammalian expression vector (Invitrogen) using jetPRIME reagent (Polyplus, Illkirch, France) according to the manufacturer's protocols. Patch-clamp measurements were performed 24 hours after transfection. For whole-cell recording, the cells were superfused with a bath solution containing $(\mathrm{mM}) 150 \mathrm{NaCl}, 5 \mathrm{KCl}, 1.6 \mathrm{CaCl}_{2} \cdot 6 \mathrm{H}_{2} \mathrm{O}$, $1.13 \mathrm{MgCl}_{2} \cdot 6 \mathrm{H}_{2} \mathrm{O} ; 1.2 \mathrm{NaH}_{2} \mathrm{PO}_{4}, 10$ glucose, 2 Na pyruvate, and 10 HEPES; the $\mathrm{pH}$ was adjusted to 7.4 with $\mathrm{NaOH}$. The patch pipettes (resistance, 3-6 $\mathrm{M} \Omega$ ) were filled with an "intracellular" solution containing $(\mathrm{mM}) 60 \mathrm{KCl}, 65 \mathrm{~K}$-glutamate, 5 EGTA, $3.5 \mathrm{MgCl}_{2}, 2 \mathrm{CaCl}_{2}, 3 \mathrm{~K}_{2} \mathrm{ATP}, 0.2 \mathrm{Na}_{2} \mathrm{GTP}, 5$ HEPES; the $\mathrm{pH}$ was adjusted to 7.2 with $\mathrm{KOH}$. Steady-state currentvoltage relations were obtained by applying slow voltage ramps $\left(40 \mathrm{mV} \mathrm{s}^{-1}\right)$ between -90 and $+30 \mathrm{mV}$. The liquid junction potential between the patch electrode and the bath solution $(\sim-8 \mathrm{mV})$ was not compensated.

For cell-attached or outside-out recording, the patch pipettes (resistance, 3-6 M $\Omega$ ) were coated with the elastomer R6101 (Dow Corning) to reduce capacitative noise; the pipettes were filled with an "intracellular" solution containing (mM) $140 \mathrm{KCl}, 5 \mathrm{HEPES}$, and $1 \mathrm{MgCl}_{2} \cdot 6 \mathrm{H}_{2} \mathrm{O}$, and the cells were superfused with a solution containing $(\mathrm{mM}) 150 \mathrm{NaCl}, 5 \mathrm{KCl}$, $1.6 \mathrm{CaCl}_{2} \cdot 6 \mathrm{H}_{2} \mathrm{O}, 1.13 \mathrm{MgCl}_{2} \cdot 6 \mathrm{H}_{2} \mathrm{O} ; 1.2 \mathrm{NaH}_{2} \mathrm{PO}_{4}, 10$ glucose, $2 \mathrm{Na}$ pyruvate, and $10 \mathrm{HEPES}$; the $\mathrm{pH}$ was adjusted to 7.4 with $\mathrm{NaOH}$. The liquid junction potential between the patch electrode and the bath solution $(\sim-8 \mathrm{mV})$ was not compensated. The sampling rate was $10 \mathrm{kHz}$; the filter had a cutoff frequency of $2 \mathrm{kHz}$. All electrophysiological experiments were carried out at room temperature $\left(20-23^{\circ} \mathrm{C}\right)$.

Voltage-Clamp Measurements with Xenopus Oocytes. For Xenopus oocyte expression studies, complementary RNA (cRNA) coding for THIK-1, TRAAK, TREK-1c, or TREK- $1 \mathrm{c}^{\mathrm{S} 344 \mathrm{~A}}$ was transcribed in vitro using T7 RNA polymerase (mMessage mMachine T7 Kit; Ambion). cRNA quality was determined by gel electrophoresis and UV spectroscopy. Defolliculated Xenopus oocytes (stage V) were injected with nuclease-free water containing cRNA of THIK1 (6 ng/oocyte), TRAAK (2.5 ng/oocyte), or TREK-1c (2.5 ng/ oocyte). The oocytes were incubated at $19^{\circ} \mathrm{C}$ for $24-48$ hours in ND96 solution containing (mM) $96 \mathrm{NaCl}, 2 \mathrm{KCl}, 1 \mathrm{MgCl}_{2}, 1.8$ $\mathrm{CaCl}_{2}$, and 5 HEPES (pH 7.5), supplemented with $100 \mu \mathrm{g} / \mathrm{ml}$ gentamycin, $2.5 \mathrm{mM}$ sodium pyruvate. For voltage-clamp recording, the oocytes were placed in a small-volume perfusion chamber and superfused with ND96 solution. Twomicroelectrode voltage-clamp measurements with rampshaped voltage commands were performed with a TurboTec$10 \mathrm{C}$ amplifier (npi electronic, Tamm, Germany). Data acquisition and analysis were performed with software developed in our laboratory (PC.DAQ1.2); data were recorded at 
A

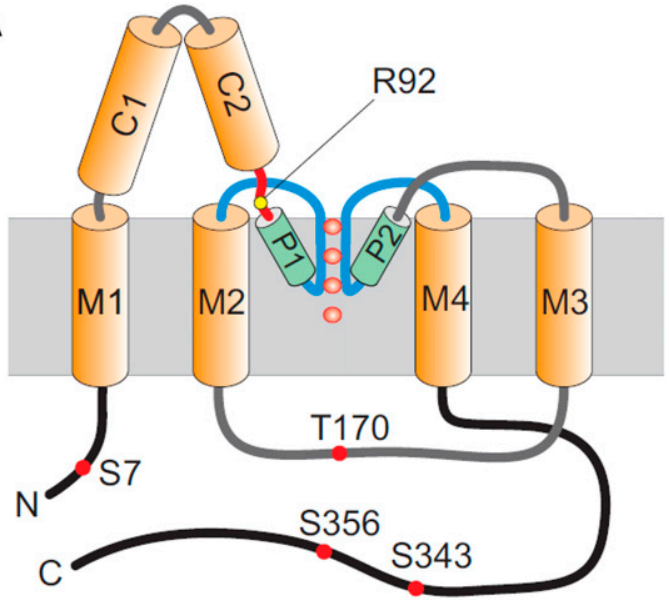

C

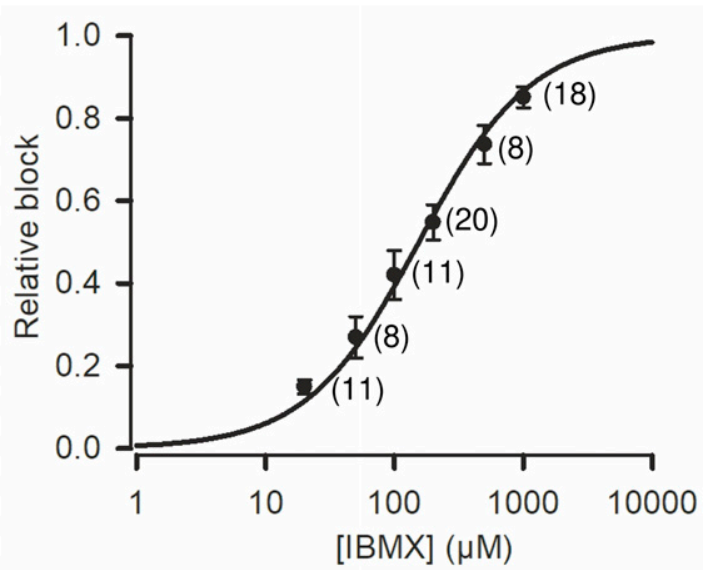

B

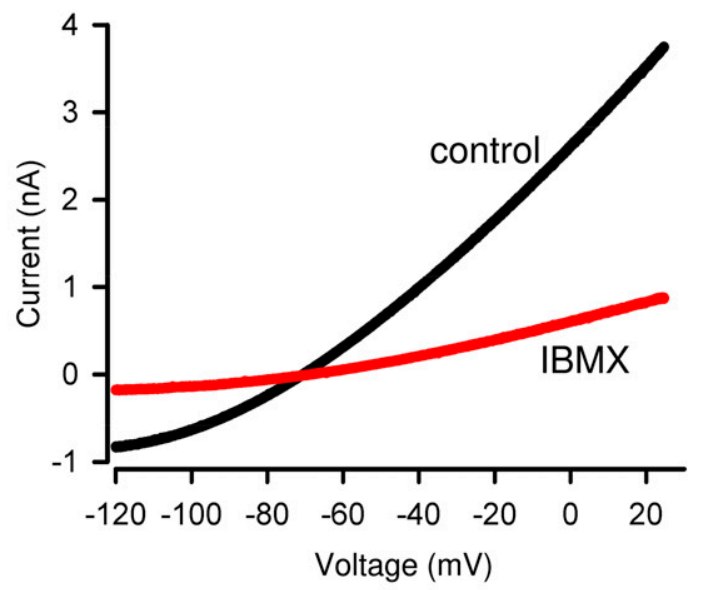

D

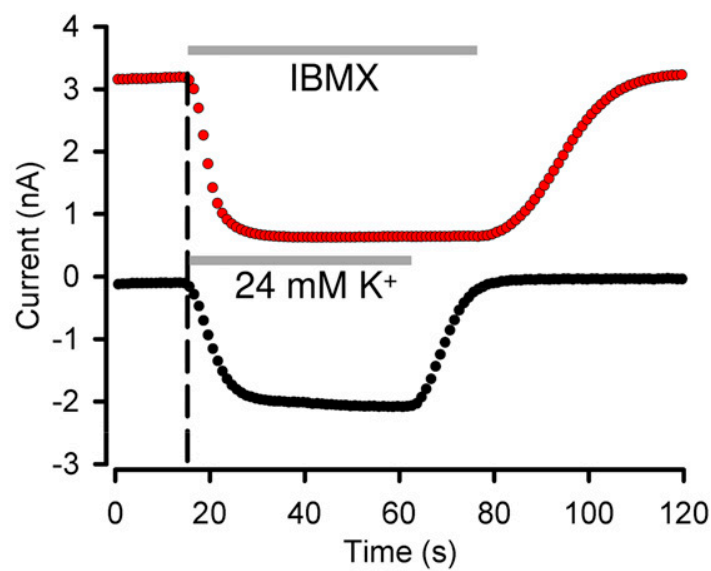

Fig. 1. The effect of IBMX on THIK-1 currents measured in CHO cells. (A) Topology of THIK-1 and localization of predicted PKA phosphorylation sites. M1, M2, M3 and M4 are the four transmembrane domains, C1 and C2 are the two helical cap domains, and P1 and P2 are the two pore domains of the channel subunit; the red spheres between $\mathrm{P} 1$ and $\mathrm{P} 2$ represent dehydrated potassium ions; the linker between $\mathrm{C} 2$ and $\mathrm{P} 1$ is shown in red. $\mathrm{N}$ and $\mathrm{C}$ are the $\mathrm{NH} 2$ and $\mathrm{COOH}$ termini of the channel subunit. (B) Typical current-voltage relations in CHO cells transfected with THIK-1, measured before (black line) and after (red line) application of $1 \mathrm{mM}$ IBMX. (C) Dose-response curve for the fractional block of THIK-1 current by IBMX; the number of CHO cells from which the data were obtained is indicated in brackets. The error bars indicate the standard deviation. (D) Red curve: typical recording of the time course of the effect of IBMX on THIK-1 outward currents measured at $0 \mathrm{mV}$. Black curve: the effect of changing the extracellular potassium concentration from 5 to $24 \mathrm{mM}$ on THIK-1 inward currents measured at $-80 \mathrm{mV}$. The two time courses (red and black curve) were recorded sequentially in the same CHO cell.

a sampling rate of $120 \mathrm{~Hz}$. For quantification of current amplitude under different experimental conditions, the currents were measured at $0 \mathrm{mV}\left(2 \mathrm{mM}\right.$ extracellular $\left.\mathrm{K}^{+}\right)$. All electrophysiological experiments were carried out at room temperature $\left(20-23^{\circ} \mathrm{C}\right)$.

Homology Modeling of THIK-1 Channels. A homology model of rat THIK-1 was constructed based on the structure of human TRAAK channels (Brohawn et al., 2012, 2013) using the Schrödinger Maestro 10.1 software package (release 20151), which includes, among others, the tools "Small Molecule Drug Discovery Suite," "Biologics Suite," "Multiple Sequence Viewer," "Prime 3.9," and "Structure Prediction Wizard." rTHIK-1, hTHIK-1, and hTRAAK were aligned using the "Multiple Sequence Viewer." In the original study of Brohawn et al. (2012), the structure of hTRAAK could be resolved from position 28 to 286 in chain A and from position 28 to 284 in chain $\mathrm{B}$, except for a flexible region unresolved for position
104-109 for chain A and 105-109 for chain B, which corresponds to the C2-P1 linker (shown in red in Fig. 1A). The structure coordinates of hTRAAK and the part of the alignment of rat THIK- 1 that covers the amino acids represented in the structure were fed into "Structure Prediction Wizard," and the final homology model was constructed using the energybased building method of Prime 3.9.

Statistics. Data are reported as means \pm S.D. Statistical significance was determined using Student's two-tailed $t$ test. Usually, the individual measurements are also shown as scatter dot plots.

\section{Results}

THIK-1 Currents are Inhibited by IBMX. The rat ortholog of the $\mathrm{K}_{2 \mathrm{P}}$-channel THIK-1 has four plausible protein kinase A (PKA) phosphorylation sites, as predicted by linear 

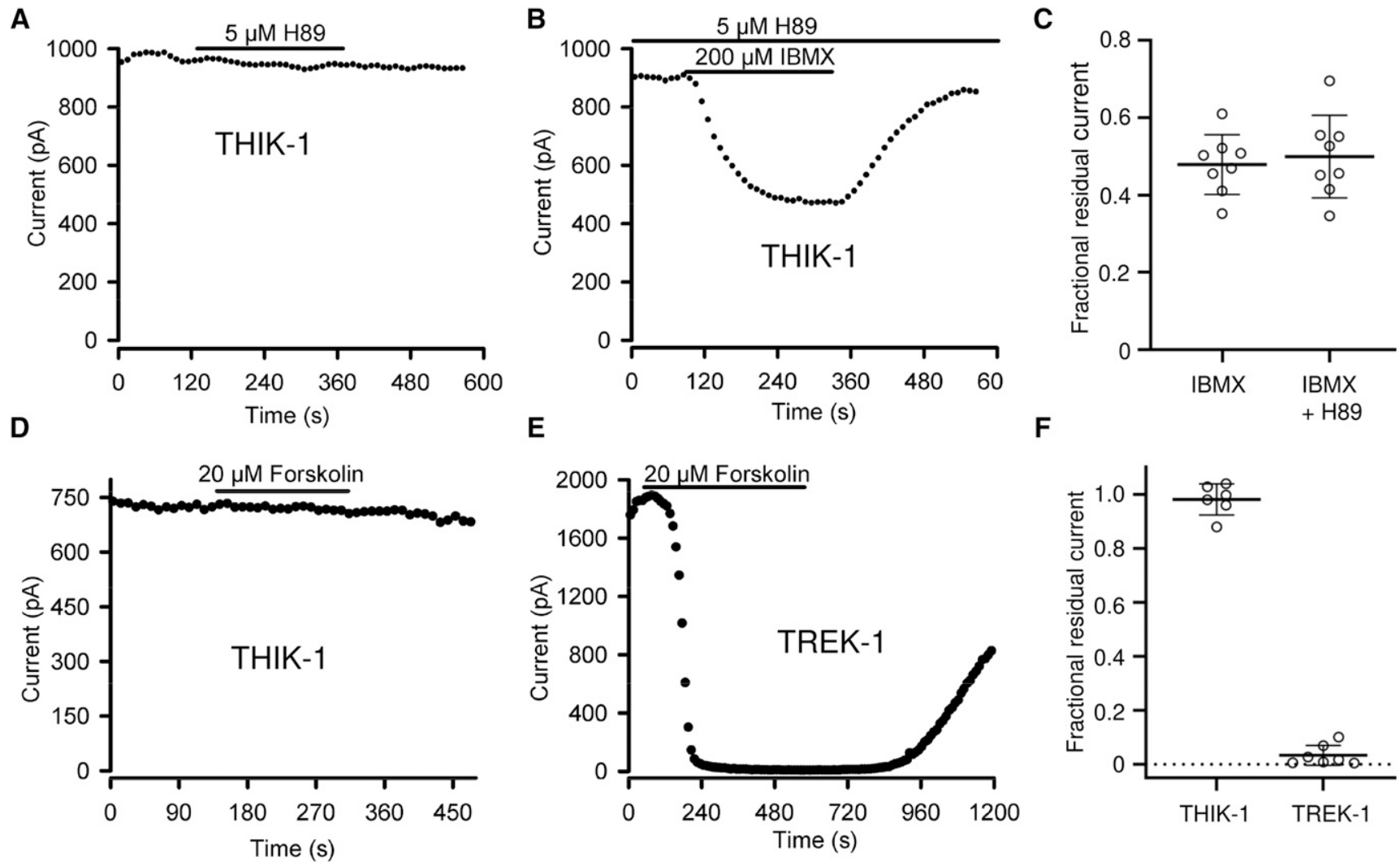

Fig. 2. The effects of the PKA inhibitor H89 and the PKA activator forskolin on THIK-1 currents were measured in CHO cells at a holding potential of $0 \mathrm{mV}$. (A) Application of $5 \mu \mathrm{M}$ H89 had no effect on whole-cell currents in CHO cells transfected with THIK-1. (B) The effect of $200 \mu \mathrm{M}$ IBMX on THIK-1 currents measured after preincubation with $5 \mu \mathrm{M}$ H89. (C) Scatter dot plot (means \pm S.D.) of the inhibitory effect of IBMX on THIK-1 currents with and without preincubation with H89. Open circles represent individual measurements (individual CHO cells). The fractional residual current measured in the presence of IBMX is shown; it is defined as $\mathrm{I}_{\mathrm{IBMX}} / \mathrm{I}_{0}$, where $\mathrm{I}_{0}$ is the current measured before application of IBMX, and $\mathrm{I}_{\text {IBMX }}$ is the current measured after application of $200 \mu \mathrm{M}$ IBMX. (D) Application of $20 \mu \mathrm{M}$ forskolin had no effect on THIK-1 currents in CHO cells. (E) Application of $20 \mu \mathrm{M}$ forskolin strongly inhibited TREK-1 currents in CHO cells. (F) Scatter dot plot (means \pm S.D.) of the effect of $20 \mu \mathrm{M}$ forskolin on THIK-1 and TREK-1 currents. The fractional residual current is shown; it is defined as $\mathrm{I}_{\text {for }} / \mathrm{I}_{0}$, where $\mathrm{I}_{0}$ is the current measured before application of forskolin and $\mathrm{I}_{\text {for }}$ is the current measured after application of $20 \mu \mathrm{M}$ forskolin.

motif analysis (NetPhosK1.0, scansite3, PhosphoELM): S7, T170, S343, and S356 (Fig. 1A). To test whether the open probability of THIK-1 channels may be modulated by PKA, we studied the effect of the phosphodiesterase inhibitor IBMX on THIK-1 current. Application of IBMX is expected to cause a rise in intracellular cAMP and a subsequent activation of endogenous protein kinase $\mathrm{A}$.

THIK-1 channels were expressed in a mammalian cell line (CHO cells), and whole-cell currents were measured using the patch-clamp technique. The cells were superfused with a physiologic salt solution containing $5 \mathrm{mM}$ extracellular potassium, and current-voltage relations were obtained using slow voltage ramps $(40 \mathrm{mV} / \mathrm{s})$. Application of $1 \mathrm{mM}$ IBMX caused a block of inward and outward currents with a reversal potential of about $-80 \mathrm{mV}$, consistent with a potassiumselective channel (Fig. 1B). The steady-state dose-response curve for the effect of IBMX on THIK-1 currents could be fitted with a Hill coefficient of 1 and an $\mathrm{IC}_{50}$ of $120 \mu \mathrm{M}$ (Fig. 1C).

The time course of the effect of IBMX on THIK- 1 currents was determined by measuring the outward current at $0 \mathrm{mV}$ at intervals of 1 second. The inhibition of outward current elicited by application of $1 \mathrm{mM}$ IBMX reached a steady state within 15 seconds; it was $90 \%$ complete within 10 seconds (Fig. 1D; red curve). The effect of IBMX was readily reversible; the increase in current during washout reached a steady state within 40 seconds (Fig. 1D). To assess the time course of the solution change in the recording chamber, we changed the extracellular potassium concentration from 5 to $24 \mathrm{mM}$ and monitored the resulting change in the inward current at $80 \mathrm{mV}$ (Fig. 1D; black curve). We found that the solution change was $90 \%$ complete within 12 seconds. These results suggest that the time constant of the binding $\left(\tau_{\mathrm{on}}\right)$ of $1 \mathrm{mM}$ IBMX to its target was too fast to be measured adequately in our recording chamber, whereas the dissociation of IBMX was somewhat slower than the solution change; the dissociation time constant of the drug $\left(\tau_{\text {off }}\right)$ is estimated to be 7 seconds or less. We conclude from these findings that the onset and washout of effects of IBMX were faster than would be expected for an intracellular site of action of the drug.

We then tried to inhibit the effect of IBMX on THIK-1 currents by preincubating the $\mathrm{CHO}$ cells for 10 minutes with the PKA inhibitor H89. We found that application of $5 \mu \mathrm{M} \mathrm{H} 89$ by itself had no effect on THIK-1 current (Fig. 2A). After preincubation of the cells with $\mathrm{H} 89$ for at least 10 minutes (Fig. 2B), the effect of IBMX was the same as without preincubation (Fig. 2C). Stimulation of adenylate cyclase by application of $20 \mu \mathrm{M}$ forskolin (which is expected to lead to activation of PKA) also had no effect on THIK-1 current (Fig. 2, D and F). As a control for the action of forskolin under our experimental conditions, we repeated these experiments with 


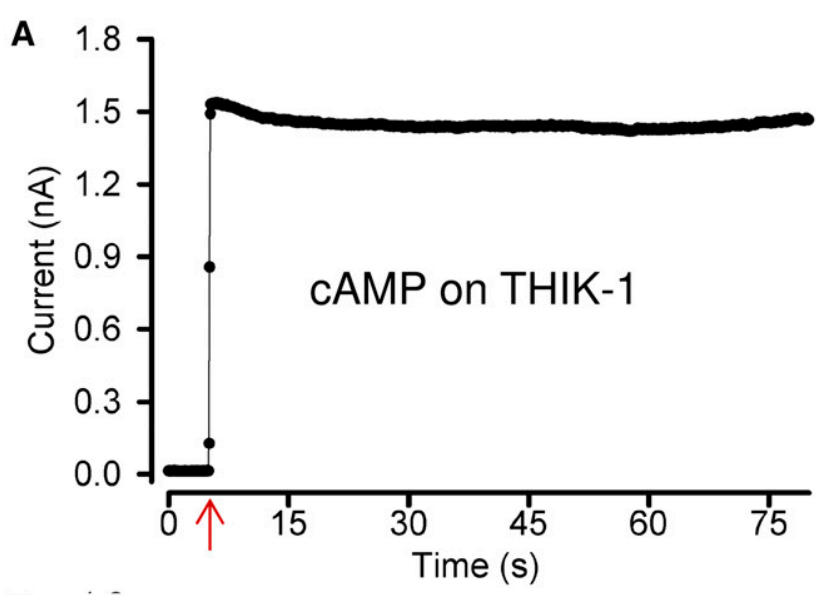

B
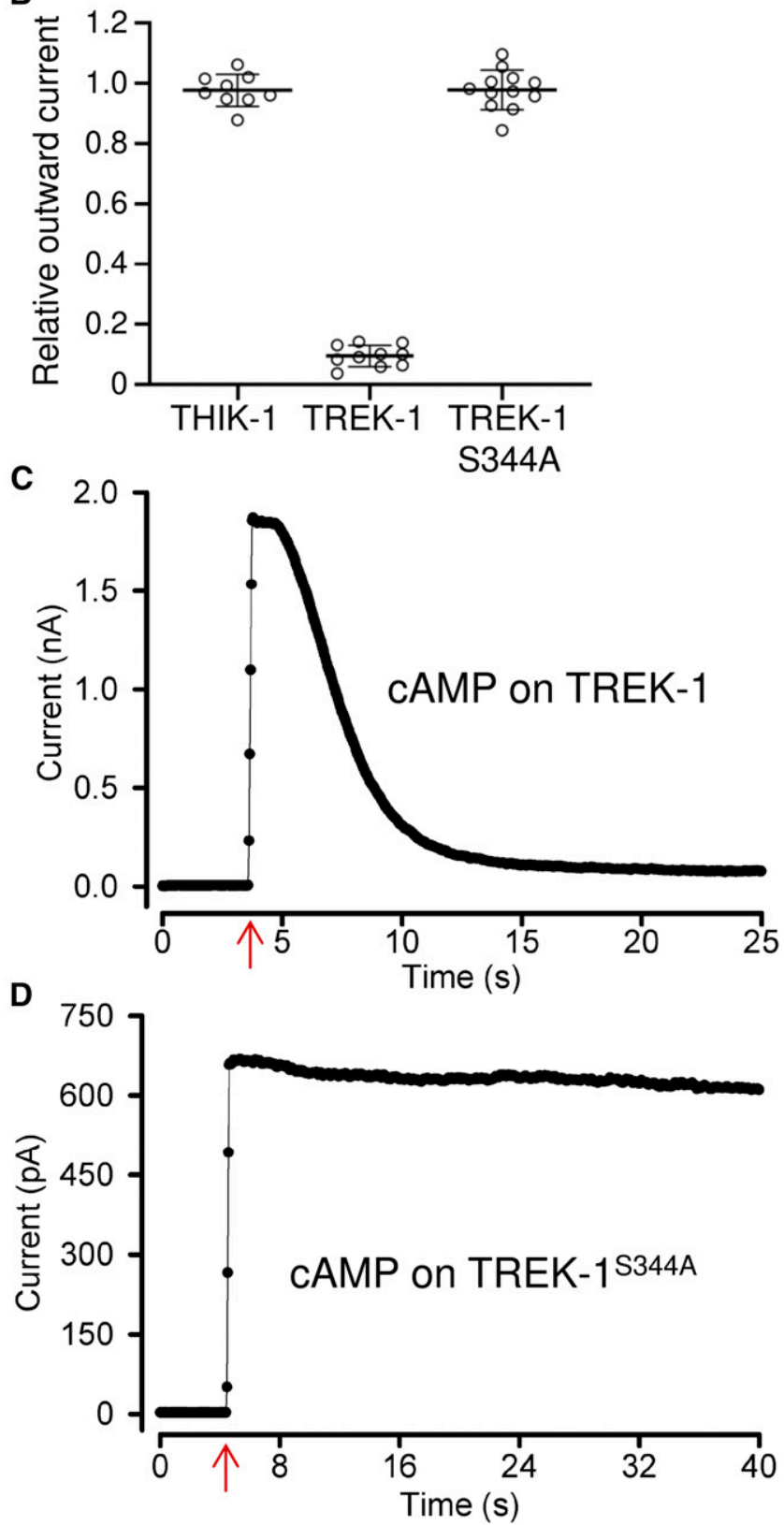

Fig. 3. The effect of intracellular application of $100 \mu \mathrm{M}$ cAMP through the patch pipette on THIK- 1 and TREK- 1 channels in CHO cells. (A) Typical measurement of the effect of intracellular cAMP on THIK-1 outward
CHO cells transfected with the $\mathrm{K}_{2 \mathrm{P}}$-channel TREK-1c (Rinné et al., 2014). Application of $20 \mu \mathrm{M}$ forskolin caused a complete and reversible inhibition of TREK-1c (Fig. 2, E and F), consistent with previous results (Fink et al., 1996; Terrenoire et al., 2001). In contrast, a mutant of TREK-1c in which the PKA phosphorylation site was removed (TREK$1 c^{\mathrm{S} 344 \mathrm{~A}}$ ) was insensitive to the action of forskolin (not illustrated).

IBMX Blocks THIK-1 Channels Only from the Extracellular Side. The results presented above suggest that TREK-1 but not THIK-1 channels can be inhibited via activation of PKA. To confirm this, we tried to apply cAMP directly to the cytosol of $\mathrm{CHO}$ cells via the pipette solution. Whole-cell recordings were carried out in CHO cells transfected with THIK-1. To induce activation of PKA, $100 \mu \mathrm{M}$ cAMP was included in the pipette solution. Currents were monitored by continuous voltage clamp at $0 \mathrm{mV}$. After formation of the gigaseal, the patch membrane was ruptured (red arrow) by applying negative pressure. Immediately after rupture of the patch, an outward current was observed that remained nearly constant as long as the cell was viable (Fig. 3, A and B), despite the fact that cAMP was allowed to diffuse into the cytosol from the patch pipette. When we repeated this experiment with CHO cells transfected with TREK-1c, a rapid decay of the outward current (recorded at $0 \mathrm{mV}$ ) was observed (Fig. 3C). A mutant of TREK-1c in which the PKA phosphorylation site at position 344 was mutated (TREK-1 $\mathrm{c}^{\mathrm{S} 344 \mathrm{~A}}$ ) was unaffected by inclusion of $100 \mu \mathrm{M}$ cAMP in the pipette solution (Fig. 3D). These experiments show that endogenous PKA in CHO cells could be activated within less than 20 seconds by dialysis of the cytoplasm with cAMP. Wild-type TREK-1c channels (but not the S344A mutant) could be inhibited by dialysis of the cells with cAMP. In contrast, THIK-1 channels were not affected by activation of PKA via cAMP. Thus, the effect of extracellular application of IBMX on THIK- 1 currents shown in Figs. 1 and 2 must be mediated by other mechanisms.

To further characterize the effect of IBMX on THIK-1 channels, we applied the drug intracellularly via the pipette solution. CHO cells were transfected with THIK-1, a gigaseal was formed, and after rupture of the patch, the cells were dialyzed with a pipette solution containing $1 \mathrm{mM}$ IBMX (Fig. 4A). The outward current at $0 \mathrm{mV}$ was constant for several minutes. We then superfused the same cell with a bath solution containing $1 \mathrm{mM}$ IBMX (Fig. 4A), and we found that the outward current was inhibited in the same way as shown in Figs. $1 \mathrm{D}$ and $2 \mathrm{~B}$. When we repeated the IBMX dialysis experiments with $\mathrm{CHO}$ cells transfected with TREK-1c, the results were quite different: the TREK-1 current was inhibited completely within 10 seconds after rupture of the patch

current in a CHO cell. The holding potential was set to $0 \mathrm{mV}$, and a cellattached patch was formed and subsequently ruptured by application of negative pressure at the time indicated by the red arrow, allowing cAMP to diffuse into the cell. (B) Scatter dot plot (means \pm S.D.) of the relative outward current $\left(I_{1} / I_{0}\right)$ measured after dialysis of the cell with cAMP; $I_{1}$ is the current measured 1 minute after rupture of the patch membrane; $I_{0}$ is the current recorded immediately after rupture of the patch. The cells were transfected with THIK-1, TREK-1c, or TREK-1 $c^{\mathrm{S} 344 \mathrm{~A}}$. (C) Typical measurement of the effect of intracellular cAMP on TREK-1 current. (D) Typical measurement of the effect of intracellular cAMP on TREK- $1 c^{\text {S344A }}$ current. 


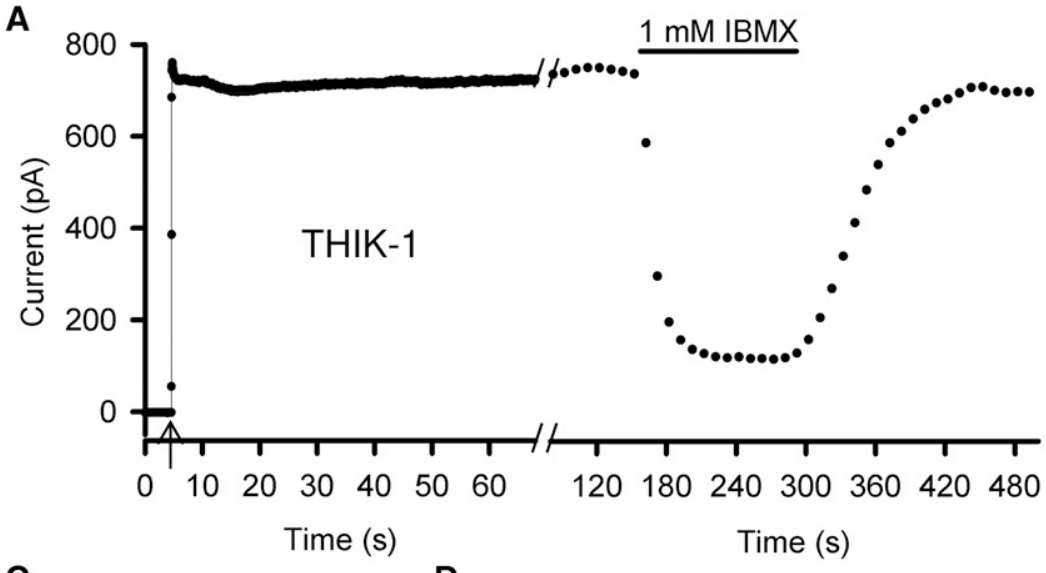

C

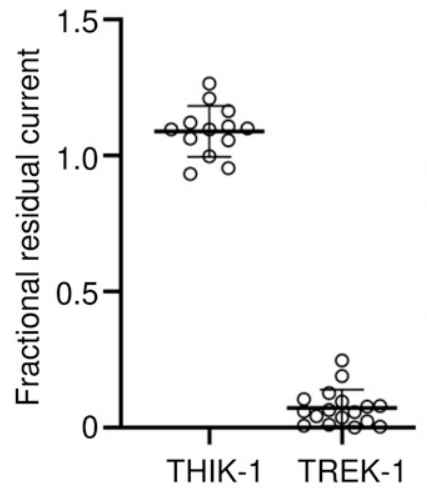

D

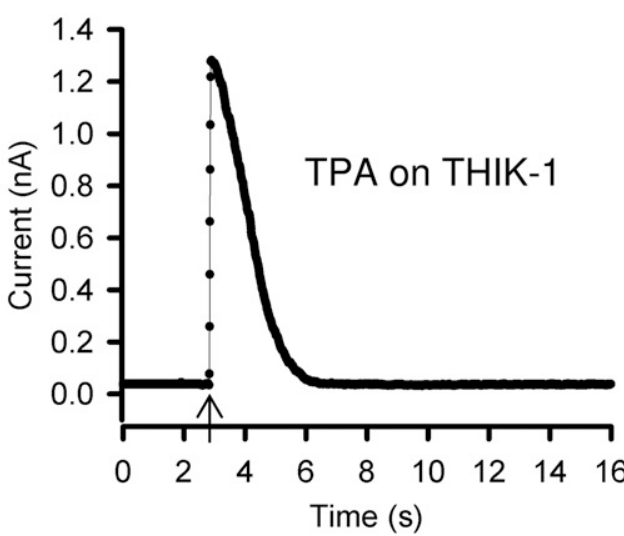

B

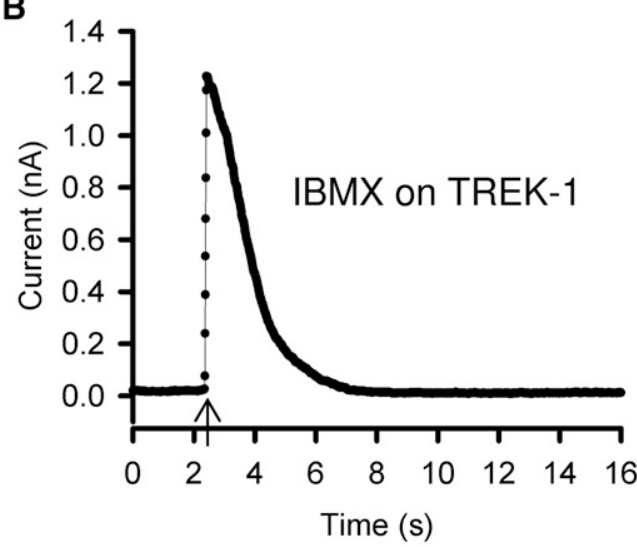

E

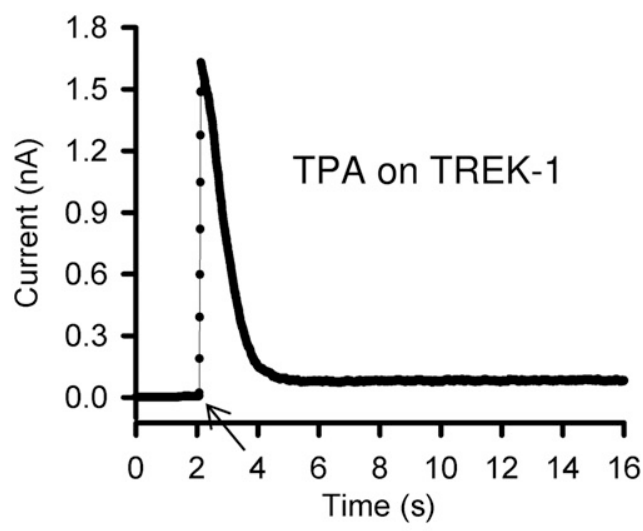

Fig. 4. The effects of intracellular and extracellular application of IBMX on THIK-1 in CHO cells, with TREK-1 channels being used as controls. (A) Application of $1 \mathrm{mM}$ IBMX via the patch pipette (left) and via the bath solution (right) in a CHO cell transfected with THIK-1 (continuous recording); please note that the time scale varies between the left and right part of the graph. The experimental procedure for intracellular drug application was the same as described in Fig. 3; the patch was ruptured at the time indicated by the arrow. (B) Application of $1 \mathrm{mM}$ IBMX via the patch pipette in a CHO cell transfected with TREK-1. (C) Scatter dot plot of fractional residual current $\left(\mathrm{I}_{\mathrm{IBMX}} / \mathrm{I}_{0}\right)$ measured 1 minute after intracellular application of $1 \mathrm{mM}$ IBMX in CHO cells transfected with THIK-1 or TREK-1. (D) Application of $20 \mu \mathrm{M}$ TPA via the patch pipette in a CHO cell transfected with THIK-1. (E) Application of $20 \mu \mathrm{M}$ TPA via the patch pipette in a CHO cell transfected with TREK-1.

(Fig. 4B). This effect was most likely due to diffusion of IBMX into the cell, inhibition of phosphodiesterases, and subsequent activation of PKA. The fractional residual current $\left(\mathrm{I}_{\mathrm{IBMX}} / \mathrm{I}_{0}\right)$ measured after dialysis of the cells with $1 \mathrm{mM}$ IBMX for 20 seconds is shown in Fig. 4C. Thus, intracellular dialysis of the cells with IBMX caused a complete block of outward current in cells transfected with TREK-1 but had no effect in cells transfected with THIK-1. Taken together, these data suggest that intracellular application of IBMX has no effect on THIK-1 channels, whereas extracellular application of IBMX causes rapid inhibition of THIK- 1 channels. Our results also imply that the permeability of the cell membrane to IBMX was not large enough to cause binding of intracellularly applied IBMX to an extracellular binding site; in other words, any IBMX appearing outside the cell was rapidly diluted by diffusion and by superfusion of the cell.

As a positive control for our experimental approach, we dialyzed the cells with the $\mathrm{K}^{+}$channel blocker tetrapentylammonium (TPA). We found that THIK-1 channels could be inhibited by intracellular dialysis with $20 \mu \mathrm{M}$ TPA, as illustrated in Fig. 4D. TREK-1c channels could also be inhibited by intracellular application of $20 \mu \mathrm{M}$ TPA (Fig. 4E), in agreement with previous results (Piechotta et al., 2011).
THIK-1 Channels in Cell-Attached and Outside-Out Patches. The results presented above suggest that IBMX blocks THIK-1 channels from the extracellular side but not from the intracellular side of the membrane. To obtain further information on the localization of the binding site for IBMX (intracellular or extracellular), we performed cell-attached and outside-out patch-clamp measurements in CHO cells transfected with THIK-1 channels. As a first step, we characterized the properties of THIK-1 channels in cell-attached patches (Fig. 5A). The cells were superfused with physiological salt solution containing $5 \mathrm{mM} \mathrm{K} \mathrm{K}^{+}$. The pipette solution contained $145 \mathrm{mM} \mathrm{K}^{+}$. The membrane potential of the cell measured after subsequent rupture of the patch had a value of $-60 \mathrm{mV}$. The current noise in the cell-attached patch was minimal at pipette potentials of about $-80 \mathrm{mV}$ (in the range 60 to $-100 \mathrm{mV}$ ), which is consistent with the estimated $\mathrm{K}^{+}$ equilibrium potential across the patch membrane. We then tried to inhibit the noise attributable to $\mathrm{K}^{+}$channels by applying tetrapentylammonium, which blocks THIK-1 channels from the inside (see Fig. 4). Figure 5B shows that the noise at -80 to $+40 \mathrm{mV}$ was markedly reduced after application of $300 \mu \mathrm{M}$ TPA in the bath solution. The concentration of TPA in the cytosol was probably much lower than $300 \mu \mathrm{M}$. 
A

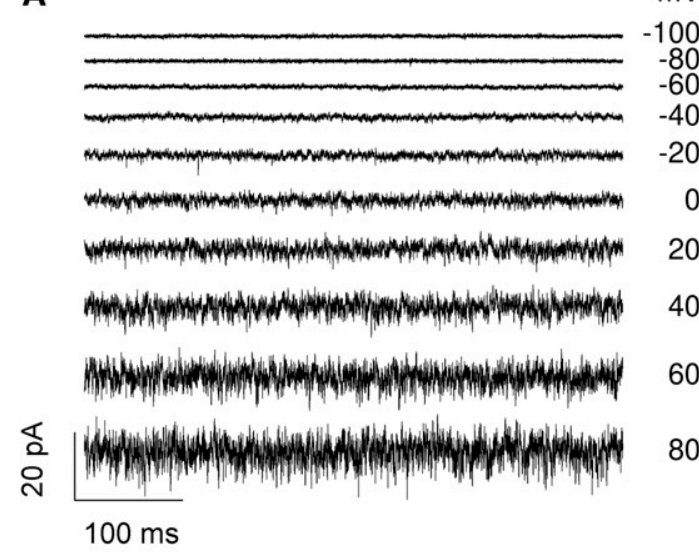

C

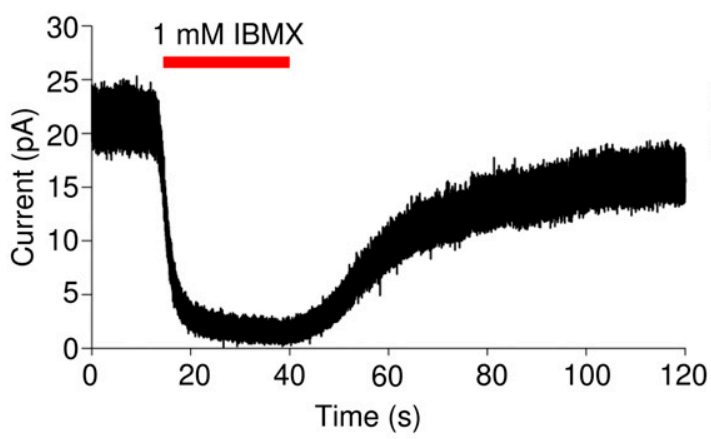

B

$\mathrm{mV}$

$-100$

$-80$

$-60$

$-40$

$-20$

0

20

40

60

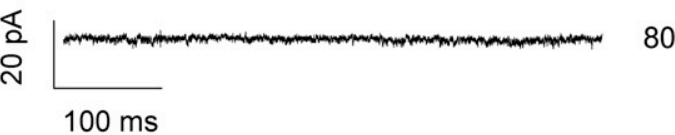

80

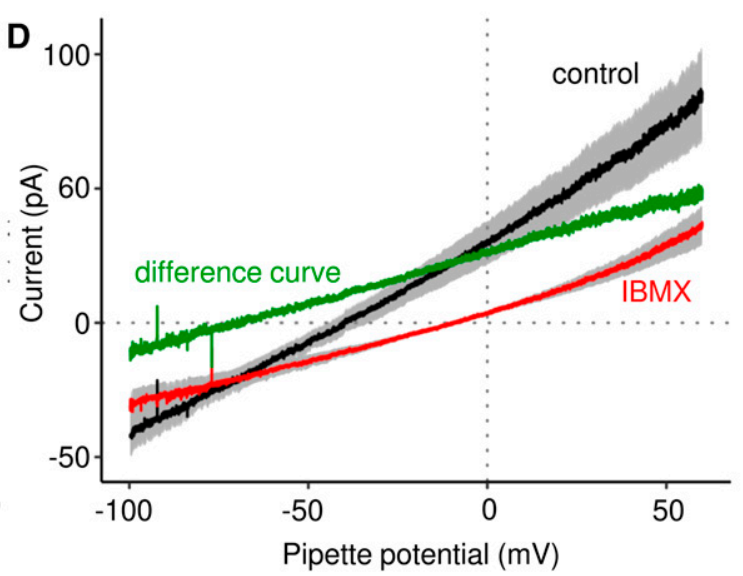

Fig. 5. THIK-1 currents in cell-attached and outside-out patches from CHO cells. The cells were superfused with physiologic salt solution containing $5 \mathrm{mM} \mathrm{K}^{+}$. (A) Typical cell-attached patch recordings at different pipette potentials (indicated on the right); the sampling rate was $15 \mathrm{kHz}$, the low-pass filter had a cutoff frequency of $2 \mathrm{kHz}$. (B) Recordings from the same patch after application of $300 \mu \mathrm{M}$ TPA to the bath solution. (C) Typical recording from an outside-out patch with $5 \mathrm{mM} \mathrm{K}^{+}$in the bath solution; the time course of the effect of $1 \mathrm{mM}$ IBMX was measured at a pipette potential of 0 mV. (D) Average current-voltage relation of outside-out patches measured before (black) and after (red) application of $1 \mathrm{mM}$ IBMX; the $95 \%$ confidence interval is indicated by the gray shading; green: the average difference curve $(n=4$ cells). The voltage on the $x$-axis corresponds to the applied pipette potential (which was not corrected for the liquid junction potential).

These results suggest that we can indeed observe an increased noise level related to THIK-1 channel activity in cell-attached patch-clamp recordings. However, the singlechannel amplitude could not be resolved, despite the fact that the noise level of our patch-clamp setup was quite low $(<200$ fA root mean square noise with a sampling rate of $10 \mathrm{kHz}$ and a low-pass filter with a cutoff frequency of $2 \mathrm{kHz}$ ). The difficulty of recording THIK-1 single-channel currents is probably related to the fact that 1) the channels have low conductance, 2) the gating kinetics of the channels is extremely fast, and 3) there were several channels in a single patch. Similar characteristics of THIK-1 channels have been reported previously (Kang et al., 2014).

Next, we carried out measurements of THIK- 1 channels in outside-out patches. The aim was to directly demonstrate the effect of extracellular IBMX in the absence of any kinase activity and in the absence of any intracellular second messengers. For these experiments, we used an extracellular (bath) solution containing $5 \mathrm{mM} \mathrm{K}^{+}$; the intracellular (pipette) solution contained $145 \mathrm{mM} \mathrm{K}^{+}$. We found that in outside-out patches, application of $1 \mathrm{mM}$ IBMX caused a rapid reduction in outward current and in current noise (Fig. 5C). This finding suggests that the patch contained several THIK-1 channels with a relatively high open probability. As in the whole-cell recordings with a rapid extracellular solution change (Fig. 1D), the binding of IBMX to the channels appeared to be faster than the dissociation. The average current-voltage relations of the outside-out patches before and after application of IBMX (Fig. 5D) crossed at about $-75 \mathrm{mV}$ (which corresponds to about $-83 \mathrm{mV}$ if the liquid junction potential of $-8 \mathrm{mV}$ is taken into account). This reversal potential is consistent with the calculated potassium equilibrium potential. Thus, our results obtained with cell-free membrane patches confirm the idea that IBMX blocks THIK-1 by binding to an extracellular domain of the channel.

The Block of THIK-1 Currents by IBMX is Not Modified by Mutation of Putative PKA Phosphorylation Sites. As a final test for the mechanism underlying the effects of IBMX, we turned to the predicted PKA phosphorylation sites (S7, T170, S343, and S356; see Fig. 1A). If mutation of one of these sites diminished the effects of IBMX on the channels, this would suggest that the inhibitory action of the drug on THIK-1 was, at least to some extent, mediated by phosphorylation of the channel protein. THIK- 1 channels 
were expressed in Xenopus oocytes, and THIK-1 currents were studied with the two-microelectrode voltage-clamp technique. When the oocytes were superfused with a bath solution containing $2 \mathrm{mM} \mathrm{K} \mathrm{K}^{+}$(ND96 solution, see Methods) the current-voltage relation of THIK-1 was outwardly rectifying (Fig. 6A), as described previously (Rajan et al., 2001). The inward and outward currents carried by THIK-1 channels were inhibited by application of $1 \mathrm{mM}$ IBMX (Fig. 6). The current change produced by IBMX reversed its polarity at approximately $-100 \mathrm{mV}$, as expected for block of a $\mathrm{K}^{+}$selective channel. We then mutated one by one all the predicted PKA phosphorylation sites: their serine or threonine residues were changed to alanine. As can be seen in Fig. 6B, the inhibition of THIK-1 by IBMX was unchanged after mutation of any of these putative phosphorylation sites. These findings suggest that the effect of IBMX on THIK-1 current was not mediated by phosphorylation of the channel protein. There are two possible explanations for the result illustrated in Fig. 6B: 1) the rise in intracellular cAMP elicited by the phosphodiesterase inhibitor IBMX and the subsequent activation of protein kinase A did not cause phosphorylation of THIK-1 channels or 2) phosphorylation of THIK-1 via PKA did not produce any measurable change in the open probability of the channels.

Identification of the IBMX Binding Site by Mutagenesis of the Helical Cap of THIK-1. To identify the binding site of IBMX on THIK-1, we mutated one by one all amino acids of the helical cap and compared the effect of IBMX on wild-type THIK-1 with the effect on the mutants (Fig. 7A). Alanine residues were mutated to serine, and all other residues were mutated to alanine. For each mutant, we calculated the fractional residual THIK-1 current during application of $500 \mu \mathrm{M}$ IBMX $\left(\mathrm{I}_{\mathrm{IBMX}} / \mathrm{I}_{0}\right)$, i.e., the ratio of the residual current measured during application of IBMX in the steady state $\left(\mathrm{I}_{\text {IBMX }}\right)$ to the current measured before application of the drug $\left(\mathrm{I}_{0}\right)$. Overall, the effect of $500 \mu \mathrm{M}$ IBMX on THIK-1 current was tested in $n=422$ oocytes ( $n=5-21$ for different mutants). During application of IBMX, most mutants showed a fractional residual current $\left(\mathrm{I}_{\mathrm{IBMX}} / \mathrm{I}_{0}\right)$ of $\sim 40 \%$, similar to wild-type THIK-1 ( $n=26$; black bar in Fig. 7A), whereas one mutant (R92A) was clearly less sensitive to inhibition by IBMX .

Figure 7A also shows that there was some scatter in the residual THIK-1 current measured during application of IBMX with different mutants. This may have been partially due to the fact that different batches of oocytes were used for different mutants; different batches showed different absolute current magnitudes. To check for the possibility of a systematic error due to variable expression levels in different oocyte batches, we plotted the absolute amplitude of the THIK-1 current against the fractional residual current (Fig. 7B). The correlation coefficient (calculated including the mutant R92A) was -0.10 , which suggests that there was practically no linear correlation between absolute THIK-1 current and the sensitivity of the channel to inhibition by IBMX. The gray lines in Fig. 7B indicate a distance of three S.D.s from the mean fractional residual current observed in the presence of IBMX. It can be seen that only one mutant, R92A, was outside this range (red circle in Fig. 7B). This makes it very unlikely that this result was obtained by chance, and it raises the possibility that this residue was somehow involved in the action of IBMX on THIK-1.

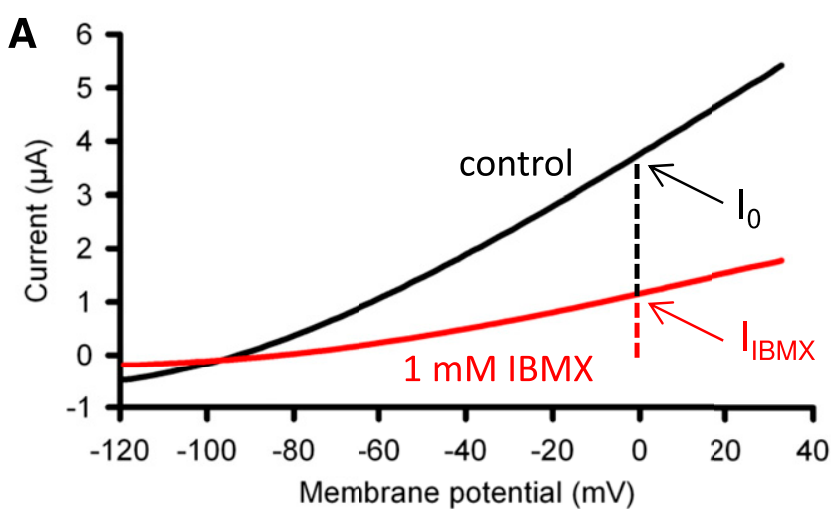

B

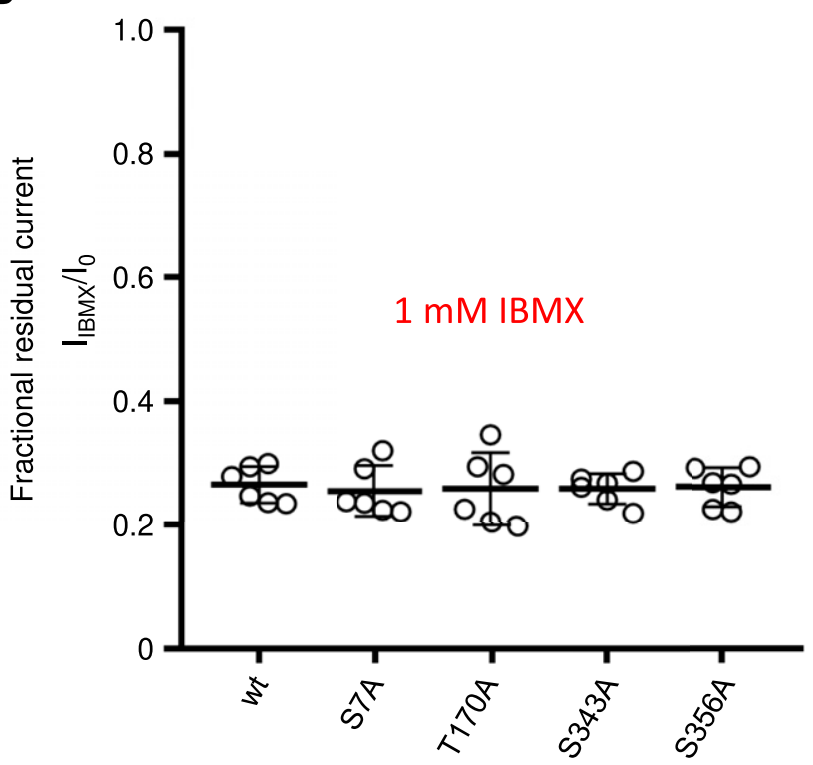

Fig. 6. The effects of IBMX on THIK-1 channels expressed in Xenopus oocytes. (A) Typical example of the current-voltage relation of THIK-1 channels in Xenopus oocytes before and after application of $1 \mathrm{mM}$ IBMX. (B) Fractional residual current $\left(\mathrm{I}_{\mathrm{IBMX}} / \mathrm{I}_{0}\right)$ measured at $0 \mathrm{mV}$ after application of $1 \mathrm{mM}$ IBMX. The oocytes were injected with cRNA coding for wild-type (wt) THIK-1 or for the THIK-1 mutants indicated.

To further confirm this hypothesis, we also mutated the residue $\mathrm{R} 92$ to glutamate and to glutamine. We found that the fractional residual current of THIK-1 current observed after application of $1 \mathrm{mM}$ IBMX was even larger in the R92E and R92Q mutants than in the mutant R92A (Fig. 7C).

The two simplest explanations for the effect of the three R92 mutations on the sensitivity of THIK-1 channels to IBMX are that 1) residue R92 is involved in the binding of IBMX to the extracellular cap of the channel or that 2) residue R92 is somehow involved in the gating of the channel. The latter effect would be expected to give rise to a change in the shape or position of the current-voltage relation. In Fig. 7D the current-voltage relations of wild-type THIK-1 and the mutants R92A, R92E, and $\mathrm{R} 92 \mathrm{Q}$ are superimposed. No change in the current-voltage relation could be detected, which makes it less likely that residue R92 is involved in the gating of the channel (see Discussion).

Figure 7E shows an alignment of the helical cap region of the $\mathrm{K}_{2 \mathrm{P}}$-channels TRAAK, TWIK-1, and TREK-2 (which have all been crystallized), together with rat THIK-1, the highly homologous rat THIK-2, and human THIK-1. It can be seen that the cap helices 1 and 2 are highly conserved in all of these 


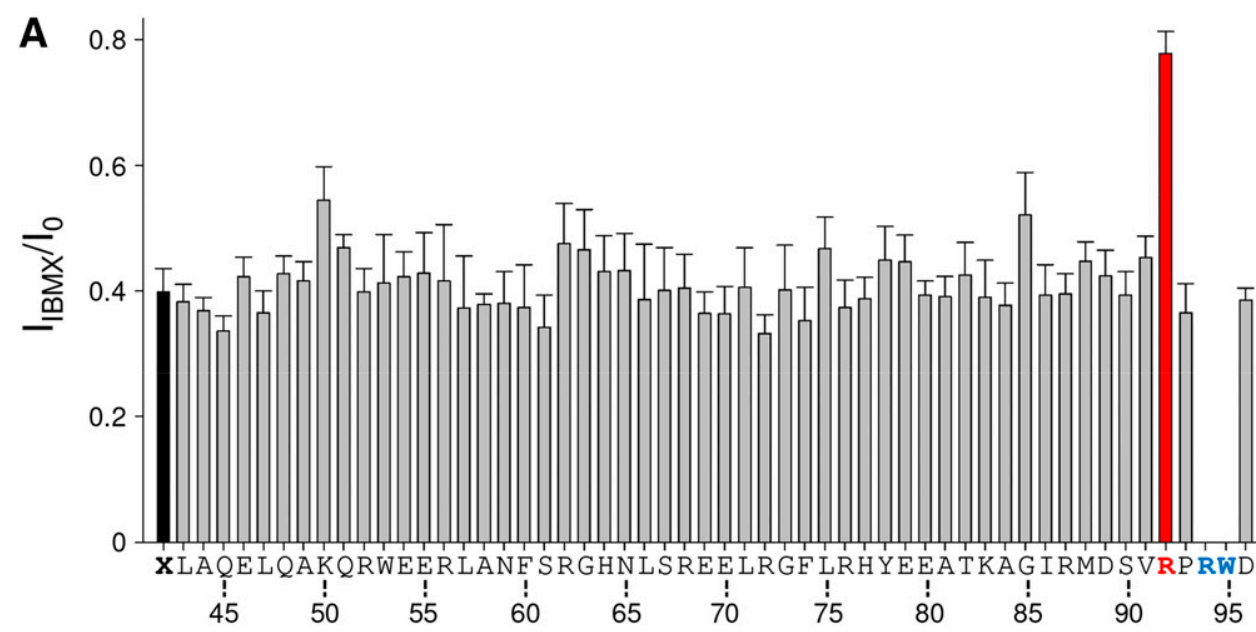

B

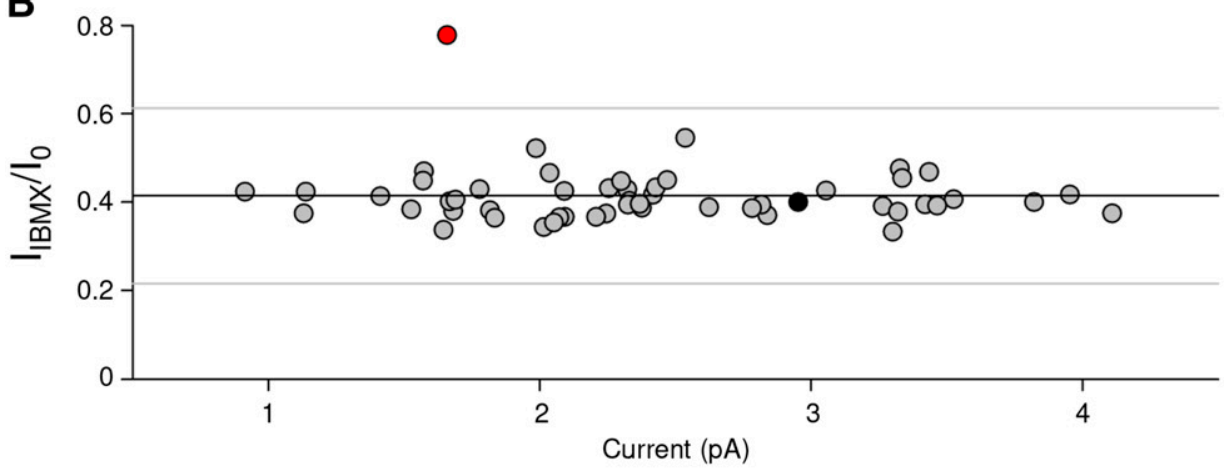

C

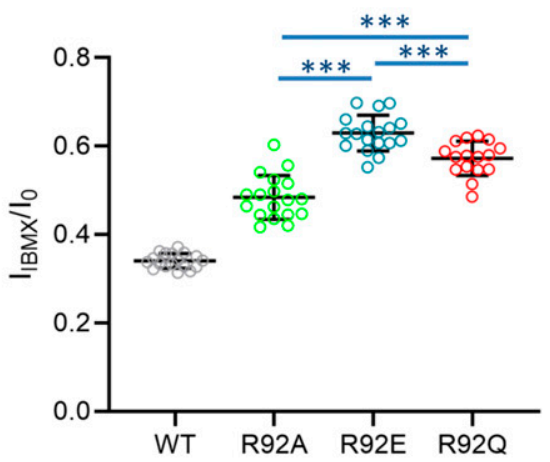

E

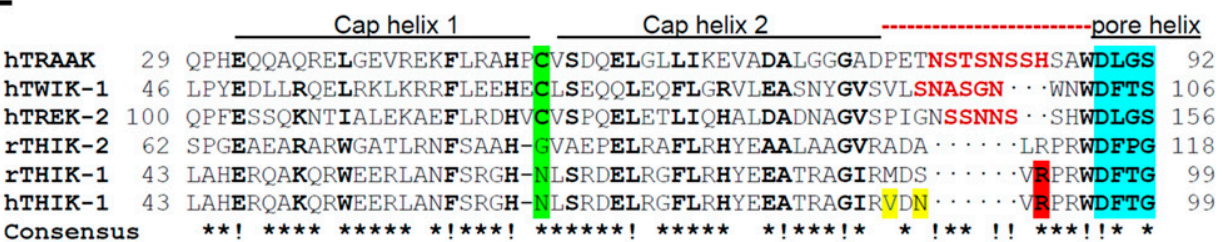

Fig. 7. Effect of mutations in the extracellular cap of rTHIK-1 on the magnitude of the inhibitory effect of IBMX on THIK-1 current in Xenopus oocytes. (A) The fractional residual THIK- 1 current $\left(\mathrm{I}_{\mathrm{IBMX}} / \mathrm{I}_{0}\right)$ measured at $0 \mathrm{mV}$ in the steady state during application of $500 \mu \mathrm{M}$ IBMX in wild-type (wt) THIK-1 and in various mutants. The residues shown on the abscissa represent the amino acids of the extracellular cap; the wild-type channel is shown in black (the bar is denoted X). The number of oocytes from which the data were obtained was 5-21 for the different mutants; the error bars represent S.D. Only one mutant (R92A, indicated in red; $n=18$ ) showed a much larger residual current (i.e., less inhibition by IBMX). Two mutants (indicated in blue) did not produce any current. (B) Plot of the absolute current measured for each mutant against the fractional residual THIK-1 current. The dark-gray horizontal line represents the mean value of $\mathrm{I}_{\mathrm{IBMX}} / \mathrm{I}_{0}$ for all mutants (including wild-type and R92A), and the light-gray lines are three S.D.s above or below the mean. The red circle indicates the mutant R92A. (C) The inhibitory effect of $1 \mathrm{mM}$ IBMX on three THIK-1 mutants with different substitutions for residue R92. (D) The shape of the current-voltage relation in different R92 mutants. Normalized current-voltage relations of wt THIK-1 (gray) and mutants R92A (green), R92E (blue), and R92Q (red); the curves were set to 1 at $0 \mathrm{mV}$. (E) Alignment of three $\mathrm{K}_{2 \mathrm{P}}$-channels whose crystal structure has been determined (TRAAK, TWIK-1, and TREK-2) as well as rat THIK-2, rat THIK-1, and human THIK-1. The residues not resolved in the crystal structure are shown in red; the cysteine residue forming the disulfide bridge between the subunits is highlighted in green; the beginning of the pore helix is highlighted in blue; the unstructured region of the $\mathrm{C} 2-\mathrm{P} 1$ linker is indicated on top by a dotted red line; conserved residues are shown in bold; residue $\mathrm{R} 92$ is highlighted in red. channels, except for the linker region (indicated by a red dashed line) between cap helix 2 (C2) and the pore helix 1 (P1). Part of the linker region was found to be disordered (Brohawn et al., 2012; Miller and Long, 2012), and it was not resolved in the crystal structure; the residues missing in the crystal structure are highlighted in red. It can be seen that the arginine residue at position 92 is at the end of the unstructured region, three residues proximal to the pore helix.

We also tested the effect of IBMX on the $\mathrm{K}_{2 \mathrm{P}}$-channel TRAAK (long Alternative Translation Initiation variant), which has a different C2-P1 linker (see Fig. 7E). We found that the current produced by TRAAK channels expressed in Xenopus oocytes was completely insensitive to extracellular application of $1 \mathrm{mM}$ IBMX ( $n=8$ oocytes; not illustrated).

Taken together, our mutagenesis experiments suggest that the "disordered" C2-P1 linker region (Brohawn et al., 2012, 2014; Miller and Long, 2012; Dong et al., 2015) may play a role in the block of THIK- 1 by IBMX. The mutations R92A, R92E, and $\mathrm{R} 92 \mathrm{Q}$ were the only ones in the entire helical cap that markedly attenuated the effect of IBMX on THIK-1 channels. 

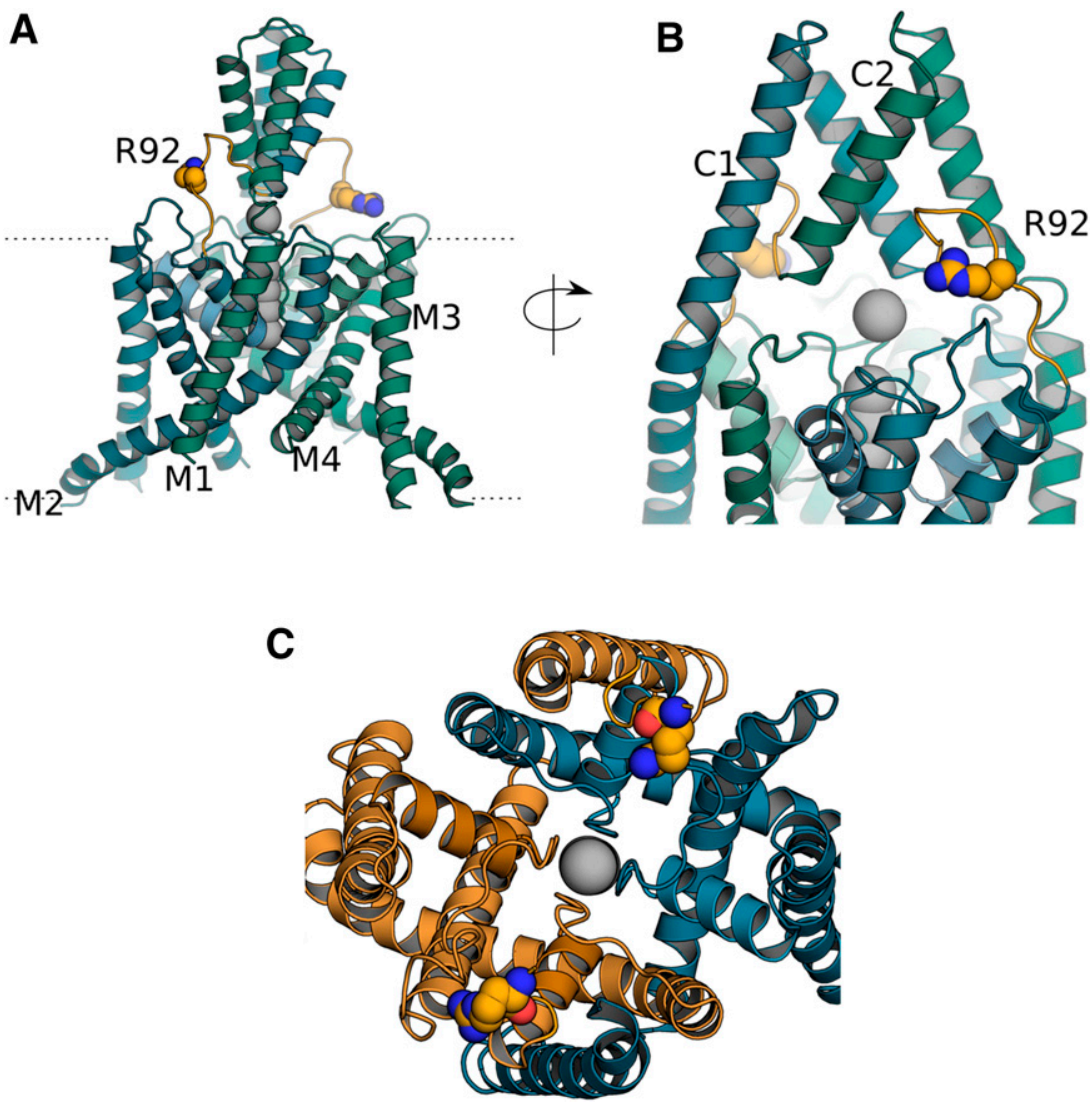

This observation is consistent with the idea that residue R92located near the end of the C2-P1 linker-may form part of the extracellular binding site of IBMX. To visualize the localization of R92, we constructed a homology model of THIK-1 (see Methods) using the TRAAK-fab structure (Brohawn et al., $2013)$ as template. Figure 8 shows that in the homology model, residue $\mathrm{R} 92$ is in the wall of the outer vestibule, not far from the outer opening of the selectivity filter.

The Effects of Other Xanthine Derivatives on THIK-1 Currents. To find out which molecular features of IBMX are involved in the binding to THIK-1, we studied the effects of some other xanthine derivatives on THIK-1 channels expressed in Xenopus oocytes and CHO cells. In both expression systems, $1 \mathrm{mM}$ theophylline had no effect, and $1 \mathrm{mM}$ caffeine had only a very weak effect (Fig. 9, A-D). Xanthine, 1methyl-xanthine, and 1,3-diethyl-7-methyl-xanthine were applied at a concentration of $100 \mu \mathrm{M}$ because of their low water solubility. These substances also had no effect on THIK-1 currents in Xenopus oocytes, whereas $100 \mu$ M IBMX reduced THIK-1 currents by approximately 20\% (Fig. 9E). Taken together, our results with various xanthine derivatives suggest that the 3-isobutyl group of IBMX is important for its inhibitory effect on THIK-1 channels; a methyl group at this position, as in theophylline, or a hydrogen atom, as in 1methyl-xanthine, does not appear to be sufficient.

\section{Discussion}

IBMX Blocks THIK-1 Channels from the Extracellular Side. Our experimental results suggest that the phosphodiesterase inhibitor IBMX acts on THIK-1 channels exclusively
Fig. 8. Homology model of THIK-1 based on the TRAAK (4I9W) crystal structure. (A) Ribbon representation viewed from within the membrane; the $\mathrm{C} 2-\mathrm{P} 1$ linker is shown in yellow; residue R92 is shown as spheres (C atoms, yellow; $\mathrm{N}$ atoms, blue). (B) View of the helical cap rotated by $90^{\circ}$ and enlarged. R92 is localized in the connecting loop between cap domain and the first pore helix. This region of the channel has not been resolved in any crystal structure of $\mathrm{K}_{2 \mathrm{P}}$-channels published so far and was modeled de novo here. (C) View from the outside into the channel cavity. All residues of the extracellular cap beyond residue R92 have been removed; R92 is shown as spheres. In all panels, the large gray spheres represent potassium ions with their hydration shells removed. from the outside and that it inhibits THIK-1 currents by binding to the helical cap of the channel. This inference is based on the following findings: 1) Extracellular application of IBMX caused a rapid and reversible block of THIK-1 currents in Xenopus oocytes (two-electrode voltage clamp) and in a mammalian cell line (whole-cell recording). The time course of the current change observed during application of IBMX was largely determined by the speed of the solution change in the recording chamber; 2) intracellular application of IBMX through the patch pipette in the whole-cell recording mode had no effect on THIK-1 currents. Intracellular application of TPA, which was used as a positive control, inhibited both THIK-1 and TREK-1 currents; 3 ) in outside-out patches from CHO cells transfected with THIK-1, application of IBMX rapidly and reversibly blocked potassium-selective channels; 4) mutation of an arginine residue in the outer cavity of THIK1 to alanine (R92A) markedly reduced the effect of IBMX on THIK-1 current.

Our discovery of the direct block of the channel from the extracellular side was fortuitous because our experiments were originally aimed at studying the modulation of THIK-1 currents via PKA using IBMX as a blocker of cAMP phosphodiesterase. Because THIK-1 has four predicted PKA phosphorylation sites (Fig. 1A), it is important to show that the effects of IBMX on THIK-1 currents described here are not in any way related to a rise in intracellular cAMP and subsequent activation of PKA. The evidence for this can be summarized as follows: 1) The effect of IBMX on THIK-1 current was unchanged after mutation of any of the four predicted PKA phosphorylation sites. 2) The effect of IBMX persisted when the cells were preincubated with the PKA 

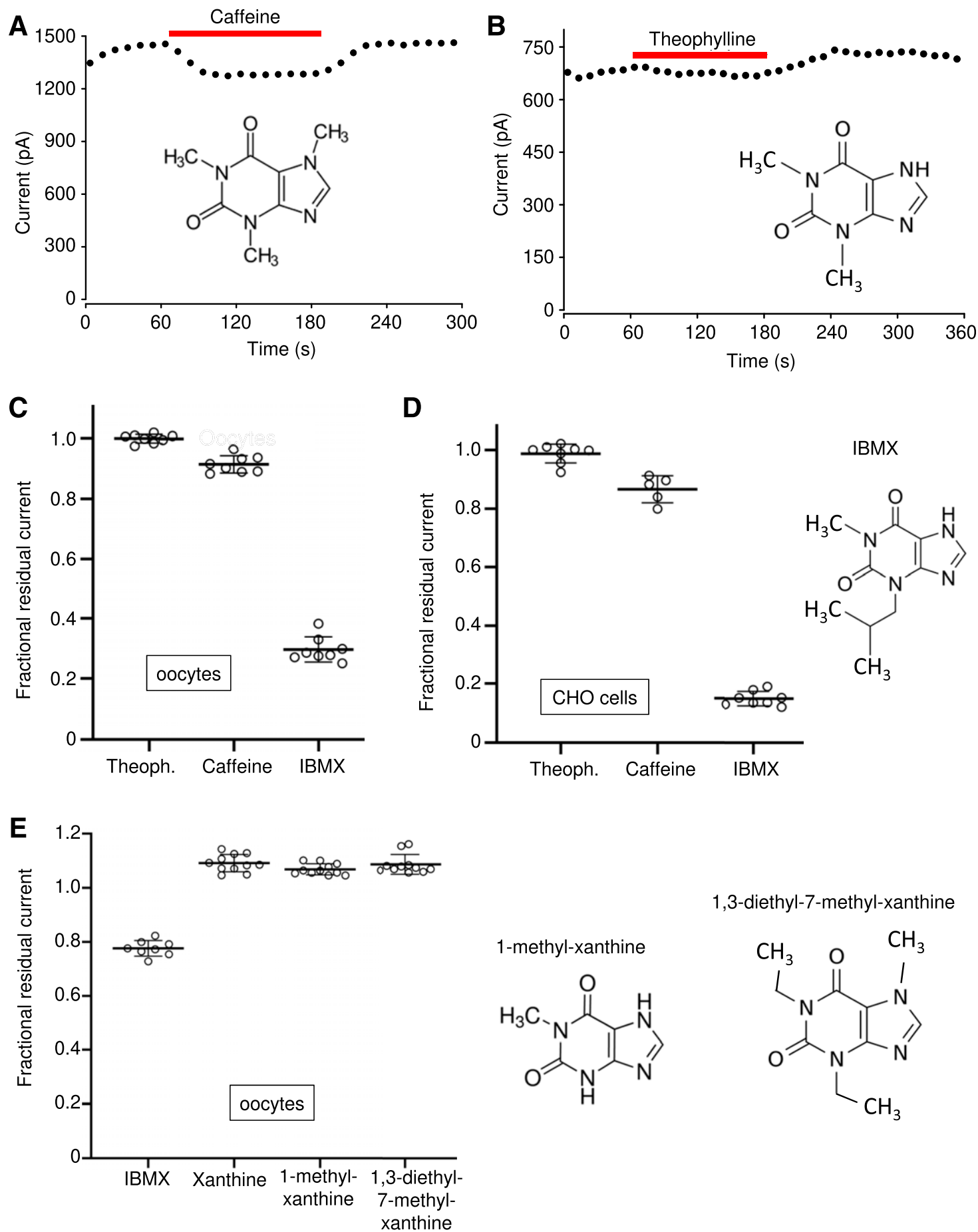

1,3-diethyl-7-methyl-xanthine

1-methyl-xanthine<smiles>Cn1c(=O)[nH]c2nc[nH]c2c1=O</smiles><smiles>CCn1c(=O)c2c(ncn2C)n(CC)c1=O</smiles>

Fig. 9. Effects of various xanthine derivatives on THIK-1 currents. Typical effects of $1 \mathrm{mM}$ caffeine (A) and $1 \mathrm{mM}$ theophylline (B) on THIK-1 currents in Xenopus oocytes. (C and D) Fractional residual THIK-1 current measured during application of $1 \mathrm{mM}$ theophylline, $1 \mathrm{mM}$ caffeine, and $1 \mathrm{mM}$ IBMX in Xenopus oocytes (C) and in CHO cells (D). (E) Fractional residual THIK-1 current measured during application of $100 \mu$ M IBMX, $100 \mu$ M xanthine, 100 $\mu \mathrm{M}$ 1-methylxanthine, and $100 \mu \mathrm{M}$ 1,3-diethyl-7-methylxanthine in Xenopus oocytes (there was a slight "run-up" of current, which explains the increase in fractional residual current).

inhibitor H89. 3) Intracellular application of cAMP through the patch pipette had no effect on THIK-1 current but inhibited TREK-1 current, which was used as a positive control. 4) Intracellular application of tetrapentylammonium blocked both THIK-1 and TREK-1 channels. 5) Application of the PKA activator forskolin had no effect on THIK-1 currents but inhibited TREK-1 currents. 6) The inhibitory effect of
IBMX on THIK-1 current persisted in outside-out patches, in which phosphorylation via PKA should be eliminated due to absence of ATP in the pipette solution.

Caffeine $(1 \mathrm{mM})$ had a much smaller inhibitory effect on THIK-1 channels than IBMX, whereas theophylline (1 mM), 1methyl-xanthine (1 mM), and 1,3-diethyl-7-metylxanthine (1 $\mathrm{mM}$ ) had no effect at all, indicating that perhaps the isobutyl 
group of IBMX may be relevant for the binding to THIK-1. Now that we have identified a seed molecule that blocks THIK-1 channels from the extracellular side of the membrane, it appears possible to start a structure-based search of specific THIK-1 modulators that do not have unwanted "side effects."

THIK-1 channels are very unusual potassium channels. In cell-attached and outside-out recordings the (voltage-dependent) noise attributable to the channel openings could be clearly seen. However, the single-channel conductance of THIK-1 could not be resolved using a sampling rate of 10 $\mathrm{kHz}$ and a bandwidth of $2 \mathrm{kHz}$. Because the root mean square noise in cell-attached or outside-out patches at the $\mathrm{K}^{+}$ equilibrium potential was $<200 \mathrm{fA}$ and the currents in the patches were between 10 and $40 \mathrm{pA}$, it may be concluded that each patch contained several channels with very short mean open times.

Furthermore, it should be noted that THIK-1 channels are robustly expressed in microglial cells and in neurons of many nuclei in the brain. Thus, experiments on these cells using IBMX or caffeine as pharmacological tools should take into account possible direct effects of IBMX on THIK-1.

Localization of the Binding Site on the Extracellular Cap. Our mutagenesis experiments analyzing the effects of IBMX on THIK-1 currents point to an important role of residue $\mathrm{R} 92$ in the $\mathrm{C} 2 \mathrm{-P} 1$ linker, which is localized to the outer cavity of the channel near the entry of the selectivity filter (Fig. 8). Of all the residues localized to the extracellular cap, only mutation of $\mathrm{R} 92$ produced a major change in the effect of IBMX on THIK-1 currents. How can we interpret the observation that mutations in $\mathrm{R} 92$ attenuated the inhibitory effect of IBMX? In principle, there are three possibilities: 1) The simplest mechanistic interpretation of our results is that R92 may form part of the IBMX binding site and that the binding of the drug to the outer cavity of the channel near the selectivity filter or to the side portal occludes the permeation of potassium ions. 2) Another possible interpretation is that binding of IBMX may have a purely allosteric effect, i.e., IBMX does not bind to R92 but elsewhere, and this may affect the gating of the channel by inducing a conformational change. The modulatory effect of the R92 mutations would then be explained by the fact that these mutations affect the gating of the channel (considering that R92 is not too far from the selectivity filter). However, it is then difficult to see where the IBMX binding site could be because none of the other mutations of the extracellular cap modulated the effect of IBMX. 3) Finally, it is conceivable that residue R92 is involved in both the binding of IBMX and in the gating at the selectivity filter, and both are affected by the mutations. In this case, the binding of IBMX would not necessarily occlude the permeation of potassium ions. At present, none of these interpretations can be excluded. The observation that the mutations did not change the shape of the current-voltage relation is consistent with the idea that R92 is involved in the binding of IBMX rather than in the gating of the channel. Structural studies of THIK-1 are required to clarify the most-likely orientations of R92.

Taken together, our results strongly suggest that some xanthine derivatives may act as extracellular blockers of THIK-1 channels. The outer cavity and/or the side portals of the extracellular cap were also found to harbor binding sites for water-soluble drugs in some other $\mathrm{K}_{2 \mathrm{P}}$-channels. The cationic dye ruthenium red was found to block TASK-3,
TREK-1, and TREK-2 channels by binding to a site within the extracellular cavity (Czirják and Enyedi, 2003; Braun et al., 2015). Another small-molecule drug, the quinoline derivative TKDC, has recently been suggested to block TREK-1 and TREK-2 channels by binding to their extracellular caps (Luo et al., 2017). Because the extracellular cap is quite unique among ion channels, and the C2-P1 linker of $\mathrm{K}_{2 \mathrm{P}^{-}}$ channels is quite variable, it may be possible to design drugs specific for extracellular binding sites of particular $\mathrm{K}_{2 \mathrm{P}^{-}}$ channels.

\section{Acknowledgments}

We thank Kirsten Ramlow and Doris Wagner for excellent technical support. The support of the High Performance and Cloud Computing Group at the Zentrum für Datenverarbeitung of the University of Tübingen is gratefully acknowledged. None of the authors has any competing interests.

\section{Authorship Contributions}

Participated in research design: Zou, Conrad, Schlichthörl, Krüger, Daut, Renigunta.

Conducted experiments: Zou, Conrad, Schlichthörl, Netz, Renigunta.

Contributed new reagents or analytic tools: Koschinsky, PreisigMüller, Krüger.

Performed data analysis: Zou, Conrad, Schlichthörl, Netz, Krüger, Daut, Renigunta.

Wrote or contributed to the writing of the manuscript: Conrad, Daut, Renigunta.

\section{References}

Aller M and Wisden W (2008) Changes in expression of some two-pore domain potassium channel genes (KCNK) in selected brain regions of developing mice. Neuroscience 151:1154-1172

Bayliss D and Barrett P (2008) Emerging roles for two-pore-domain potassium channels and their potential therapeutic impact. Trends Pharmacol Sci 29 $566-575$.

Bichet D, Blin S, Feliciangeli S, Chatelain F, Bobak N, and Lesage F (2015) Silent but not dumb: how cellular trafficking and pore gating modulate expression of TWIK1 and THIK2. Pflugers Arch 467:1121-1131.

Braun G, Lengyel M, Enyedi P, and Czirják G (2015) Differential sensitivity of TREK-1, TREK-2 and TRAAK background potassium channels to the polycationic dye ruthenium red. Br J Pharmacol 172:1728-1738.

Brohawn S, Campbell E, and MacKinnon R (2013) Domain-swapped chain connectivity and gated membrane access in a Fab-mediated crystal of the human TRAAK $\mathrm{K}^{+}$channel. Proc Natl Acad Sci USA 110:2129-2134.

Brohawn S, Campbell E, and MacKinnon R (2014) Physical mechanism for gating and mechanosensitivity of the human TRAAK $\mathrm{K}^{+}$channel. Nature 516:126-130. Brohawn S, del Mármol J, and MacKinnon R (2012) Crystal structure of the human $\mathrm{K}_{2 \mathrm{P}}$ TRAAK, a lipid- and mechano-sensitive $\mathrm{K}^{+}$ion channel. Science 335:436-441. Butovsky O, Jedrychowski M, Moore C, Cialic R, Lanser A Gabriely G, Koeglsperger T, Dake B, Wu P, Doykan C, et al. (2014) Identification of a unique TGF- $\beta$-dependent molecular and functional signature in microglia. Nat Neurosci 17 131-143.

Chatelain F, Bichet D, Feliciangeli S, Larroque M, Braud V, Douguet D, and Lesage $\mathrm{F}$ (2013) Silencing of the tandem pore domain halothane-inhibited $\mathrm{K}^{+}$channel 2 (THIK2) relies on combined intracellular retention and low intrinsic activity at the plasma membrane. J Biol Chem 288:35081-35092.

Czirják G and Enyedi P (2003) Ruthenium red inhibits TASK-3 potassium channel by interconnecting glutamate 70 of the two subunits. Mol Pharmacol 63:646-652.

Dong Y, Pike A, Mackenzie A, McClenaghan C, Aryal P, Dong L, Quigley A, Grieben M, Goubin S, Mukhopadhyay S, et al. (2015) K2P channel gating mechanisms revealed by structures of TREK-2 and a complex with Prozac. Science 347: $1256-1259$.

Duprat F, Lauritzen I, Patel A, and Honoré E (2007) The TASK background K2P channels: chemo- and nutrient sensors. Trends Neurosci 30:573-580.

Enyedi P and Czirják G (2010) Molecular background of leak $\mathrm{K}^{+}$currents: two-pore domain potassium channels. Physiol Rev 90:559-605.

Feliciangeli S, Chatelain F, Bichet D, and Lesage F (2015) The family of $\mathrm{K}_{2 \mathrm{P}}$ channels: salient structural and functional properties. J Physiol 593:2587-2603.

Fink M, Duprat F, Lesage F, Reyes R, Romey G, Heurteaux C, and Lazdunski M (1996) Cloning, functional expression and brain localization of a novel unconventional outward rectifier $\mathrm{K}^{+}$channel. EMBO J 15:6854-6862.

Girard C, Duprat F, Terrenoire C, Tinel N, Fosset M, Romey G, Lazdunski M, and Lesage F (2001) Genomic and functional characteristics of novel human pancreatic $2 \mathrm{P}$ domain $\left.\mathrm{K}^{+}\right)$channels. Biochem Biophys Res Commun 282:249-256.

Kang D, Hogan J, and Kim D (2014) THIK-1 (K2P13.1) is a small-conductance background $\left.\mathrm{K}^{+}\right)$channel in rat trigeminal ganglion neurons. Pflugers Arch 466 $1289-1300$ 
Lazarenko R, Fortuna M, Shi Y, Mulkey D, Takakura A, Moreira T, Guyenet P, and Bayliss D (2010) Anesthetic activation of central respiratory chemoreceptor neurons involves inhibition of a THIK-1-like background $\left.\mathrm{K}^{+}\right)$current. J Neurosc 30:9324-9334.

Lesage F and Barhanin J (2011) Molecular physiology of pH-sensitive background $\mathrm{K}(2 \mathrm{P})$ channels. Physiology (Bethesda) 26:424-437.

Luo Q, Chen L, Cheng X, Ma Y, Li X, Zhang B, Li L, Zhang S, Guo F, Li Y, et al. (2017) An allosteric ligand-binding site in the extracellular cap of K2P channels. Nat Commun 8:378.

Madry C, Kyrargyri V, Arancibia-Carcamo I, Jolivet R, Kohsaka S, Bryan R and Attwell D (2018) Microglial ramification, surveillance, and interleukin-1 $\beta$ release are regulated by the two-pore domain $\mathrm{K}^{+}$channel THIK-1. Neuron $\mathbf{9 7}$ 299-312.e6.

Miller A and Long S (2012) Crystal structure of the human two-pore domain potassium channel K2P1. Science 335:432-436.

Piechotta P, Rapedius M, Stansfeld P, Bollepalli M, Ehrlich G, Andres-Enguix I, Fritzenschaft H, Decher N, Sansom M, Tucker S, et al. (2011) The pore structure and gating mechanism of $\mathrm{K}_{2 \mathrm{P}}$ channels [published correction appears in $E M B O \mathrm{~J}$ (2011) 30:4515]. EMBO J 30:3607-3619.

Rajan S, Wischmeyer E, Karschin C, Preisig-Müller R, Grzeschik K, Daut J, Karschin A, and Derst C (2001) THIK-1 and THIK-2, a novel subfamily of tandem pore domain $\mathrm{K}^{+}$channels. J Biol Chem 276:7302-7311.

Renigunta V, Schlichthörl G, and Daut J (2015) Much more than a leak: structure and function of $\mathrm{K}_{2}$ p-channels. Pflugers Arch 467:867-894.
Renigunta V, Zou X, Kling S, Schlichthörl G, and Daut J (2014) Breaking the silence: functional expression of the two-pore-domain potassium channel THIK-2. Pflugers Arch 466:1735-1745.

Rinné S, Renigunta V, Schlichthörl G, Zuzarte M, Bittner S, Meuth S, Decher N, Daut J, and Preisig-Müller R (2014) A splice variant of the two-pore domain potassium channel TREK-1 with only one pore domain reduces the surface expression of full-length TREK-1 channels. Pflugers Arch 466:1559-1570.

Terrenoire C, Lauritzen I, Lesage F, Romey G, and Lazdunski M (2001) A TREK-1like potassium channel in atrial cells inhibited by beta-adrenergic stimulation and activated by volatile anesthetics. Circ Res 89:336-342.

Yu F and Catterall W (2004) The VGL-chanome: a protein superfamily specialized for electrical signaling and ionic homeostasis. Sci STKE 2004:re15.

Zhang Y, Chen K, Sloan S, Bennett M, Scholze A, O'Keeffe S, Phatnani H, Guarnieri P, Caneda C, Ruderisch N, et al. (2014) An RNA-sequencing transcriptome and splicing database of glia, neurons, and vascular cells of the cerebral cortex. $J$ Neurosci 34:11929-11947.

Address correspondence to: Jürgen Daut, Institute of Physiology and Pathophysiology, Marburg University, Deutschhausstr. 2, 35037 Marburg, Germany. E-mail: jdaut@staff.uni-marburg.de; or Dr. Vijay Renigunta, Institute of Physiology and Pathophysiology, Marburg University, Deutschhausstr. 2, 35037 Marburg, Germany. E-mail: vijay@staff.uni-marburg.de 